\title{
New Perspectives in Underlying Molecular Defects Based Cystic Fibrosis Therapeutics
}

\author{
Rajendra Prasad ${ }^{1}$
}

Published online: 15 June 2021

(C) Association of Clinical Biochemists of India 2021

Cystic fibrosis (CF, MIM \# 219,700), an autosomal genetic disorder of chloride $\left(\mathrm{Cl}^{-}\right)$conductance across epithelial cells owing to mutations in the cystic fibrosis transmembrane conductance regulator gene (CFTR, ABCG2 MIM = 602,421) associated with various clinical phenotypes i.e. respiratory obstruction, pancreatic insufficiency, meconium ileus and male infertility due to congenital bilateral absence of the vas deferens (CBAVD, MIM \# 22,180) [1]. The Prevelance of CF varies among different ethnicity. Strikingly exact prevelance of $\mathrm{CF}$ in Indian population is not exactly known [2]. The advances in clinical care are made possible in part by the improved understanding of CF pathophysiology. Hitherto, CF treatment was based on controlling symptomatic complications owing to the loss of CFTR protein function [3]. Interestingly, in recent years the attention was turned to precise medicine for customizing individual patient driven therapies to improve outcome [4] and the development of treatments targeting the consequences of the underlying defect [5].

The CFTR gene spans across an approximately $240 \mathrm{~kb}$ region on chromosome 7q31.3 consists of 27 exons [6], which transcribed into a mature RNA of $6.5 \mathrm{~kb}$. The transcribed protein is glycosylated transmembrane protein as a member of the ABC transporter super family. Hydropathy plot analysis revealed that CFTR protein composed of two motifs, each containing a membrane spanning domain (MSD) that composed of six transmembrane helices and

Rajendra Prasad

fateh1977@yahoo.com

1 Department of Biochemistry, MM Institute of Medical Science and Research, MM (Deemed To Be University), Mullana, Ambala, Haryana 133207, India nucleotide binding domain (NBD) interacting with the ATP [7]. MSD-NBD motifs are linked by unique domain termed as regulatory domain containing multiple phosphorylation sites which catalyzed by protein kinase A and C. CFTR transporter function as a cAMP regulated chloride channel in exocrine epithelia [8]. To date, more than 2000 disease causing and benign CFTR mutations have been characterized [8]. Further these mutations were classified into six major categories based on the basis of the molecular mechanisms of each mutation contributing to CFTR dysfunction [9]. This classification system is more relevant to therapeutic strategies aimed at restoring normal CFTR function. These six classes include as follows: class-I. No synthesis of CFTR protein due to nonsense mutations i.e. G542X, T1282X, R553X etc. Class-II Mutations led to misfolding or improper processing of CFTR protein undergo degradation i.e. $\triangle F 508$, N1303 K, R $560 \mathrm{~T}$ etc. Notably, $\Delta \mathrm{F} 508$ due to 3 base pair deletion resulted in exclusion of $\Delta \mathrm{F} 508$. Class-III mutations resulted in defective chloride channels due to aberrant ATP binding and hydrolysis i.e. G551D, G178R, G551S. Class-IV mutations led to defective chloride conductance as well as its pore i.e. R117 H, R 347P, R117X.et. class-V mutations produced lesser amount of functional protein i.e. $3840+10 \mathrm{kbC}>\mathrm{T}, 3120+\mathrm{IG} \rightarrow \mathrm{A}, 2789+5 \mathrm{G} \rightarrow \mathrm{A}$. class-VI mutations caused reduction in turn-over or half-life of the abnormal CFTR protein i.e. 4326 del TC, Q1422X, 4279 Ins A [10].

Since the discovery and identification of spectrum of mutations in CFTR gene, there has been worldwide interest to correct misfolded $\Delta \mathrm{F} 508$ protein to permit transport to the surface and to activate $\mathrm{Cl}^{-}$channel function by development of pharmacological molecules. $\Delta \mathrm{F} 508$ belongs to class-II defect which are found in $90 \%$ of $\mathrm{CF}$ patients which is associated with synthesis of immature 
CFTR protein with rapid protein degradation and minimal localization at plasma membrane [4].

Recent advances of targeted molecular therapies and high throughput screening have resulted in multiple drug therapies that target many important mutations in CFTR protein. The development of pharmacological agents that target the specific defects in the CFTR protein. These molecules are classified into two categorised. Potentiators (ivacaftor: VX-770, VRT-532, PG01, SF-03); correctors (lumacaftor: VX-809, VX-661 Corr-4a etc.). The Correctors assist in the folding of mutated CFTR protein to form the right 3-D structure so that it is able to translocate to the plasma membrane. On the other hand, potentiators help in restoring cAMP dependent chloride channel activity of mutant CFTR. Recent findings demonstrated that combination therapy of corrector (VX-809) and potentiators (VX770) restore the functional activity of $\Delta F 508$ - CFTR channel to great extent. Notwithstanding, these modulators are not able to restore all functional defects of $\Delta \mathrm{F} 508$ mutation [10]. Of course, this mutation insensitive to cAMP stimulation and less stable in the cell membrane, therefore other molecules Viz amplifiers and inhibitors along with correctors and potentiators are needed to restore maximal functional activity of mutated CFTR proteins: US food and drug administration approved correctors and potentiators are in phase 2 and phase 3 clinical trials. which revealed that combination therapy of corrector and potentiator in $\Delta \mathrm{F} 508$ homozygous patients showed an inspiring result with a reduction in CF pulmonary exacerbation and improvement in sweat chloride levels. Notably, the use of these pharmacological molecules in all the types of CFTR mutations patients are in progress. At Indian perspective, there are very rare mutations so called orphan mutations which cannot provide sufficient information with regard to $\mathrm{CF}$ phenotypes. Therefore, cellular and functional information are utmost important on these mutations which can improve CF genetic counselling [10-12].

The rapid progress over the past decade in CF modulators viz corrector and potentiator therapy for $\mathrm{CF}$ is hopefully the most exciting recent advance in the field, which provide the possibility of a treatment that correct the underlying CFTR disfunction in the appropriate target cells. Strikingly the limited efficacy of first-generation correctors and potentiators as well as also lack of understanding of their mechanism of action. Therefore, it urgently warrants further basic and clinical research. In addition, there is a need of formulation and identification of safe and potent CFTR modulators with high efficacy in normalization of $\Delta$ F508-CFTR cellular processing. New screening paradigms with special reference to specific, well characterized defect in $\triangle$ F508-CFTR folding and structure, may be needed. A combination corrector therapy, for instance compound targeted to the $\Delta$ F508-CFTR protein, in combination with the agents that after the cellular proteostasis machinery. There is also a need for in vitro preclinical assays for molecular action. Novel therapeutics in CF have been transformative, demonstrating how a commitment to basic science can translate into therapeutic advances.

\section{References}

1. Welsh MJ, Ramsey BW, Accurso F, Cutting G. Cystic fibrosis. In. Scriver CR, Beaudet AL, Sly WS, Valle D. The Metabolic and Molecular Basis of Inherited Diseases. 2001:5121-88.

2. Sharma N, Singh M, Kaur G, Thapa BR, Prasad R. Identification and characterization of CFTR gene mutations in Indian CF patients. Ann Hum Genet. 2009;73(1):26-33.

3. Cohen-Cymberknoh M, Shoseyov D, Kerem E. Managing cystic fibrosis: strategies that increase life expectancy and improve quality of life. Am J Respir Crit Care Med. 2011;183(11):1463-71.

4. Clancy JP, Jain M. Personalized medicine in cystic fibrosis: dawning of a new era. Am J Respir Crit Care Med. 2012;186(7):593-7.

5. Ong T, Ramsey BW. Update in cystic fibrosis 2014. Am J Respir Crit Care Med. 2015;192(6):669-75.

6. Zielenski J, Rozmahel R, Bozon D, Kerem BS, Grzelczak Z, Riordan JR, Rommens J, Tsui LC. Genomic DNA sequence of the cystic fibrosis transmembrane conductance regulator (CFTR) gene. Genomics. 1991;10(1):214-28.

7. Riordan JR. Assembly of functional CFTR chloride channels. Annu Rev Physiol. 2005;17(67):701-18.

8. Cystic Fibrosis centre at the Hospital for sick children in Toronto. Cystic fibrosis mutation database [updated 2011, Apr. 25,] Available from http://www.genet.sickkids.on.ca.

9. Welsh MJ, Smith AE. Molecular mechanisms of CFTR chloride channel dysfunction in cystic fibrosis. Cell. 1993;73(7):1251-4.

10. Sharma H, Souchet MJ, Callebaut I, Prasad R, Becq F. Function, pharmacological correction and maturation of new Indian CFTR gene mutations. J Cyst Fibros. 2015;14(1):34-41.

11. Heltshe SL, Cogen J, Ramos KJ, Goss CH. Cystic fibrosis: the dawn of a new therapeutic era. Am J Respir Crit Care Med. 2017;195(8):979-84.

12. Sharma N, Acharya N, Singh SK, Singh M, Sharma U, Prasad R. Heterogenous spectrum of CFTR gene mutations in Indian patients with congenital absence of vas deferens. Hum Reprod. 2009;24(5):1229-36.

Publisher's Note Springer Nature remains neutral with regard to jurisdictional claims in published maps and institutional affiliations. 